\title{
¿Qué pueden aportar actualmente las nuevas tecnologías al trastorno bipolar?
}

\author{
Pilar Sierra ${ }^{1,2}$, Elisa Gallach ${ }^{1}$, Humberto Echevarría ${ }^{3}$, Ana García Blanco ${ }^{1,2}$ y Lorenzo Livianos ${ }^{1,2}$ \\ ${ }^{1}$ Hospital Universitario y Politécnico La Fe, Valencia, España \\ ${ }^{2}$ Universidad de Valencia, Valencia, España \\ ${ }^{3}$ Unidad de Salud Mental Vall de Uxó, Castellón, España
}

\begin{abstract}
Resumen: La aplicación de las nuevas tecnologías de la información y la comunicación (TIC) a la salud ha experimentado un gran auge a lo largo de la última década. Por sus características clínicas y evolutivas, el trastorno bipolar es una de las enfermedades mentales en las que su introducción es factible y aspectos claves como la psicoeducación o la adherencia terapéutica, se pueden ver beneficiados a través de estas técnicas. La posibilidad del uso de sensores abre una nueva perspectiva en la identificación de marcadores biológicos en la detección de síntomas tempranos de recaída. En el presente trabajo realizamos una revisión de las intervenciones previas en el trastorno bipolar utilizando las TIC, analizamos las ventajas y desventajas inherentes a su uso y planteamos futuras líneas de investigación que incluyan ensayos aleatorizados, que avalen su eficacia y eficiencia, junto con una mejor definición de la población diana.
\end{abstract}

Palabras clave: Trastorno bipolar; mSalud; e-salud aplicaciones; teléfonos móviles; tecnología.

What can new technologies offer to bipolar disorder at present?

\begin{abstract}
Over the last decade, the application of new information and communication technologies (ICT) to health has greatly increased. Because of its clinical and developmental characteristics, bipolar disorder is one of the mental illnesses for which its introduction is feasible and key aspects such as psychoeducation or therapeutical adherence can be enhanced through these techniques. The possibility of using sensors opens a new perspective as to identifying biological markers to detect early symptoms of relapse. In this paper we review previous interventions for bipolar disorder using ITC, we analyze benefits and disadvantages attached to their use and propose future research in this field, to include randomized trials for better efficacy and efficiency, together with a better definition of the target population.
\end{abstract}

Keywords: Bipolar disorder; mHealth; E-health applications; mobile phones; technology.

\section{Introducción}

A lo largo de la última década hemos asistido a un sorprendente crecimiento y difusión de las nuevas tecnologías de la información y la comunicación (TIC); tradi-

Recibido: 8 mayo 2015; aceptado: 29 noviembre 2015.

Correspondencia: Pilar Sierra San Miguel, Hospital Universitario la Fe, Avda. Fernando Abril Martorell 106. Edificio B, planta 7 ${ }^{\mathrm{a}}, 46026$ Valencia, España. Correo-e: sierrasanmiguel@hotmail.com Agradecimientos: Esta investigación ha sido apoyada en parte por la beca "Río Hortega" (CM14/00012) a Ana C. García-Blanco del Instituto Carlos III (Ministerio español de Economía e Innovación) cionalmente países como Australia, Suecia y Países Bajos han sido líderes en su aplicación a la salud mental (Burns, Davenport, Durkin, Luscombe y Hickie, 2010). Los analistas predicen que el teléfono móvil será la primera herramienta para la conexión a Internet en 2020 y la ubicuidad de los dispositivos personales sugiere que será factible incluirlos en la atención a la salud mental (Price et al., 2014). Actualmente gran parte de la población que acude a nuestros servicios, ha crecido utilizando de un modo natural las nuevas tecnologías. Recientemente ha publicado el informe EIU: "El paciente al poder: Cómo la tecnología móvil está transformando la salud", elaborado por The Economist Intelligence Unit. 
Su elaboración se ha prolongado durante un año, han participado 144 países y han intervenido líderes de la sanidad pública y privada, de farmacéuticas, biotecnología y servicios médicos, junto con personal técnico. La mayoría de los encuestados consideraban que las TIC acrecientan el acceso a la información médica a los pacientes, mejoran notablemente el pronóstico en salud (64\%) y favorecen la toma de decisiones sobre la salud (63\%), pudiendo reducir costes para el propio paciente (24\%) e instituciones $(17 \%)$, así como prevenir epidemias.

Las primeras aplicaciones móviles (apps) aparecieron a finales de 2008 y actualmente se estiman en más de millón y medio, siendo aproximadamente 40.000 las relacionadas con el ámbito de la salud de las cuales, el 6\% corresponden a salud mental y el $18 \%$ a temática sanitaria como control y autogestión de sueño, estrés o relajación. Sin embargo, solo una minoría de ellas está apoyada por la evidencia científica y su eficacia y eficiencia aún están en entredicho (Cohn, 2012). La FDA (Food and Drug Administration), el organismo regulador en materia de medicamentos y salud de Estados Unidos, recientemente ha hecho público un comunicado manifestando que no intervendrá de forma directa en la regulación de apps sanitarias o en el software que recopile información para dispositivos de uso médico (FDA, 2015). De este modo se trata de potenciar el desarrollo de nuevas TIC y evitar la interferencia de legislaciones restrictivas.

En líneas generales estas intervenciones se refieren al uso de teléfonos móviles o PDAs que permiten al paciente utilizar aplicaciones software para monitorizar de modo longitudinal aspectos como las variaciones del humor, el sueño o la toma de medicación. Posibilitan la psicoterapia telefónica, videoconferencias e intervenciones a través de internet. Simon y Ludman (2009), las categorizan en función del grado de interacción humana. En el nivel más alto se sitúan aquellas que suponen una comunicación directa con el terapeuta, como la psicoterapia telefónica o videoconferencia. En el nivel intermedio, figuran las sincrónicas, con mensajes de texto que aparecen en función de la presencia de determinados síntomas y que permiten pasar a un nivel máximo de interacción (contactando directamente con el paciente). O bien comunicación de mínimo nivel, de tipo asincrónico como mensajes de texto con recordatorios de fármacos pero de forma anónima. Otra posibilidad es el uso de sensores localizados en el dispositivo móvil (sistemas de dispositivos externos de posicionamiento global o GPS, bluetooth, acelerómetros) o dispositivos externos con conexiones inalámbricas (sensores de ritmo cardiaco o respuesta galvánica de la piel).
Las TIC se han utilizado con éxito en la esquizofrenia (Kimhy et al., 2006), trastornos de la conducta alimentaria (Smyth et al., 2007 o trastornos límite de la personalidad (Trull et al., 2008). También se ha demostrado su eficacia en el manejo del estrés psicológico (Serino et al., 20014). En el caso del trastorno bipolar, su uso puede resultar especialmente útil por su evolución cíclica, y por la sintomatología interepisódica subsindrómica, que resulta decisiva en los niveles de calidad de vida (Marange11 et al., 2009). Por otra parte a pesar de que las guías actuales recomiendan la combinación de psicoterapias dirigidas específicamente al trastorno bipolar con la farmacoterapia (Goodwin, 2009), sigue siendo poco frecuente que éstas formen parte de la práctica rutinaria en las consultas. En general son tratamientos prolongados demasiado costosos en tiempo o en personal o bien no existe personal entrenado adecuadamente. También los gráficos vitales han sido un método tradicional muy útil para el registro del estado clínico y evolución (Livianos, Pino y Sierra, 2003). Sin embargo el hecho de que la recogida de información se lleve a cabo de modo manual en papel, supone un obstáculo cuando las visitas son muy espaciadas o cuando los pacientes viajan lejos. Existe cada vez mayor evidencia de que las TIC representan un recurso potente y factible para la gestión de trastornos psicológicos en dos sentidos: a través del apoyo al tratamiento básico y mejorando el registro inmediato del estado psicológico (Wade, 2010).

Al margen del motivo económico, otros como las dificultades de transporte también influyen negativamente en la aplicación de intervenciones psicosociales en el trastorno bipolar (Kilbourne, 2005).

El objetivo de nuestro trabajo es revisar los estudios llevados a cabo en personas con trastorno bipolar, utilizando las nuevas TIC y analizar las ventajas y desventajas de este tipo de técnicas en la práctica diaria, centrándonos de un modo más detenido en el uso de móviles.

\section{Método}

Se realizó una búsqueda en las bases de datos PsycInfo, EMBASE, Medline y SCOPUS (2000-enero 2016). Los términos de búsqueda fueron: ("bipolar disorder","trastorno bipolar" OR "manic depressive illness", "enfermedad maniaco depresiva" OR "mania”, "manía”) AND ("mHealth" OR "e-Health", "aplicaciones E-health" OR "mobile phones", "teléfonos móviles"). Se obtuvieron inicialmente 574 publicaciones. Al mismo tiempo se realizó un análisis adicional a través de una búsqueda manual y se localizaron un total de 10 artículos que fueron añadidos. Se incluyeron estudios publicados en inglés o castellano a partir del año 2000 hasta enero del año 2016. Dado 
que se trata de un tema de reciente interés incluimos estudios piloto, aleatorizados controlados doble o simple ciego, así como estudios abiertos y descriptivos, junto con revisiones e informes en páginas web del tema de interés.

Tras revisar el título o el abstract se desecharon 372, en su mayoría por hacer referencia al uso de TICs en otras enfermedades médicas no aplicables al trastorno bipolar. Se revisó el texto completo de 47 trabajos y se excluyeron 15 , por no cumplir estrictamente los criterios de búsqueda.

En la Figura 1 se detalla el diagrama de búsqueda.

En cuanto a las características de los estudios, 4 eran estudios aleatorizados controlados, 6 longitudinales, 8 descriptivos, 5 revisiones sobre el tema, 5 estudios piloto y 4 informes en páginas web.

En la Tabla 1 se detallan las características de los estudios longitudinales y en la Tabla 2 de los aleatorizados controlados, incluidos en la revisión.

\section{Resultados}

Los resultados se dividieron en varios subapartados. En primer lugar se definen conceptos generales y a continuación se describen las intervenciones previas con TIC en el trastorno bipolar.

\section{¿Qué es la eHealth y la mHealth?}

La Organización Mundial de la Salud (OMS) define la eHealth como "El empleo de información y tecnologías de comunicación para un mejor control de la salud. Por ejemplo, para el tratamiento de determinados pacientes, fomentar la investigación, crear herramientas para la educación de estudiantes, hacer screening en diversas enfermedades y en fin, para la supervisión de la salud pública" (OMS, 2015). El concepto de telemedicina se utiliza en general para designar la prestación de servicios de medicina a distancia. Para su implementación se utilizan TIC. Incluye muchas posibilidades como consultas telefónicas con profesionales médicos, historias clínicas electrónicas, receta digital, análisis de ingentes datos sanitarios (Big Data), sistemas digitales de cribado de pacientes, programas de apoyo de decisiones clínicas, o programas de educación médica (Bird, 1975). En cuanto a la mHealth (mobileHealth), es la práctica de la medicina basada en el uso de dispositivos móviles como teléfonos móviles y PDAs (Asistente Digital Personal). Constituye un subsegmento de la salud electrónica. La recolección de datos requiere de un dispositivo de recogida de información (teléfono, ordenador o dispositivo portátil) y el software que la alberga (Cipresso, 2012).

\section{¿Qué son los BITs?}

Las BITs (Behavioral Intervention Technologies) suponen la aplicación de estrategias de evaluación e intervención conductual y psicológica, a través del uso de nuevas tecnologías para abordar objetivos conductuales, cognitivos y afectivos y así mejorar la salud física

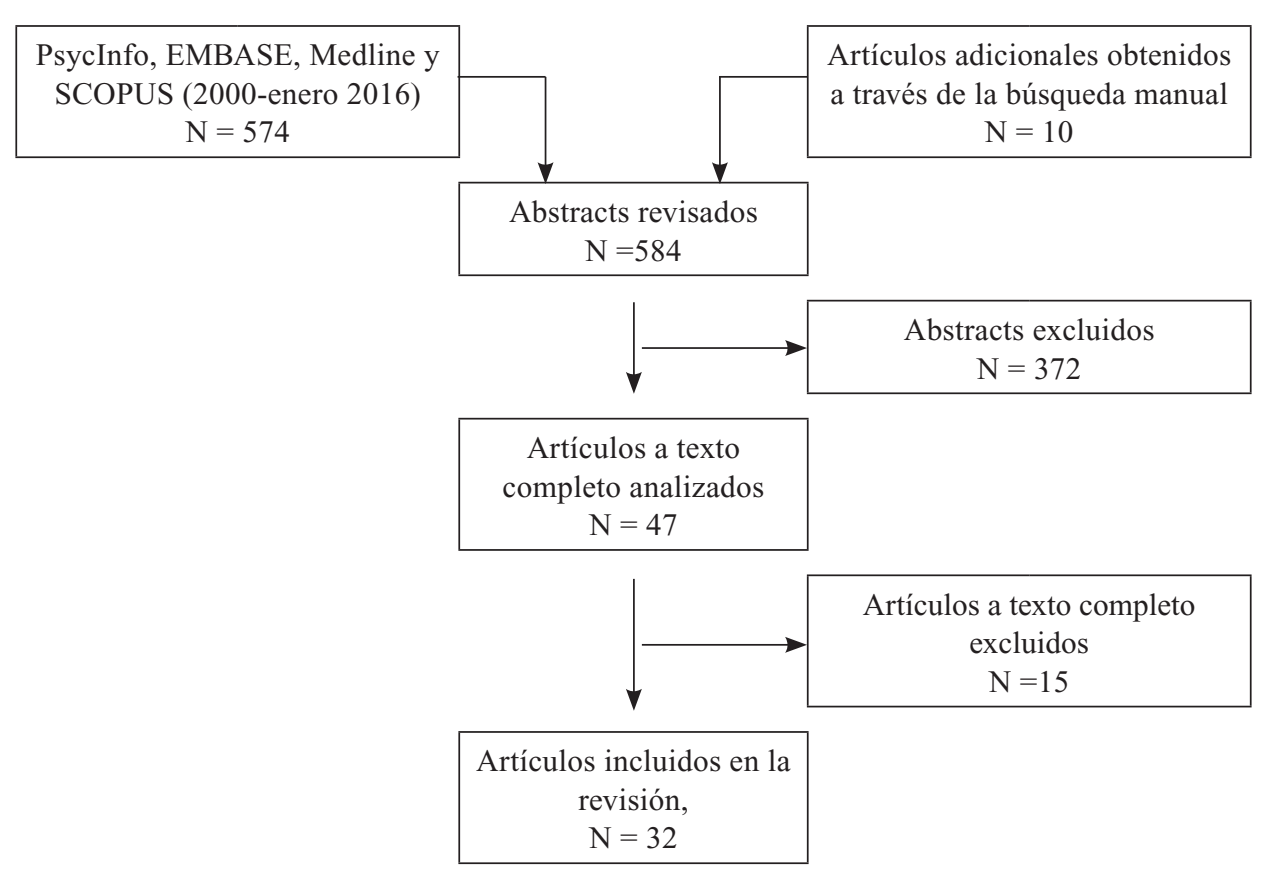

Figura 1. Diagrama de flujo de la búsqueda bibliográfica y proceso de selección de los estudios. 
Tabla 1. Características generales de los estudios longitudinales incluidos

\begin{tabular}{|c|c|c|c|c|}
\hline $\begin{array}{c}\text { Autor, año } \\
\text { publicación, país }\end{array}$ & Programa & $\begin{array}{l}\text { Tamaño de la muestra, } \\
\text { diagnósticos }\end{array}$ & Seguimiento & Resultados \\
\hline $\begin{array}{l}\text { Bauer et al., } 2004 \\
\text { Canadá \&Alemania }\end{array}$ & ChronoRecord & $\begin{array}{c}80 \\
\text { TB I }\end{array}$ & 3 meses & $\begin{array}{l}\text { Alta correlación Escala HAMD-ChronoRecord } \\
\text { Alta aceptación de los pacientes }\end{array}$ \\
\hline $\begin{array}{l}\text { Bauer et al., } 2008 \\
\text { USA, Canadá \& } \\
\text { Alemania }\end{array}$ & ChronoRecord & $\begin{array}{l}107 \\
\mathrm{~TB}\end{array}$ & 3 meses & Alta correlación Escala YMRS - ChronoRecord \\
\hline $\begin{array}{l}\text { Bopp et al., } 2010 \\
\text { USA \& UK }\end{array}$ & Mensajes texto & TB I/II: 47/15 & 36 semanas & $\begin{array}{l}\text { Resultados similares a métodos tradicionales de } \\
\text { recogida de datos }\end{array}$ \\
\hline $\begin{array}{l}\text { Depp et al., } 2010 \\
\text { USA }\end{array}$ & Programa PRISM & $\begin{array}{l}10 \\
\mathrm{~TB}\end{array}$ & 2 semanas & Alta adherencia y participación \\
\hline $\begin{array}{l}\text { Faurholt-Jepsen et } \\
\text { al., } 2014 \\
\text { Dinamarca }\end{array}$ & $\begin{array}{l}\text { Programa } \\
\text { MONARCA }\end{array}$ & $\begin{array}{c}17 \\
\text { TB I }\end{array}$ & 3 meses & $\begin{array}{l}\text { Correlación significativa puntuaciones en la } \\
\text { HAMD y nivel de actividad física }\end{array}$ \\
\hline $\begin{array}{l}\text { Scharer et al., } 2015 \\
\text { Alemania }\end{array}$ & $\begin{array}{l}\text { App gráfico vital, } \\
\text { PDA }\end{array}$ & $\begin{array}{l}54 \\
\mathrm{~TB}\end{array}$ & 18 meses & $\begin{array}{l}\text { Validez del gráfico vital en el reconocimiento } \\
\text { de episodios maníacos y depresivos }\end{array}$ \\
\hline
\end{tabular}

Nota. Escala Escala YMRS = Escala Young de manía; HAMD = Escala Hamilton de Depresión; Programa PRISM: Personalized Real Time Intervention for Stabilizing Mood; Programa MONARCA (MONitoring, treAtment and pRediCtion of bipolar disorder episodes); TB: Trastorno Bipolar; TB I: Trastorno Bipolar tipo I; TB II: Trastorno Bipolar tipo II.

Tabla 2. Características generales de los estudios aleatorizados controlados

\begin{tabular}{|c|c|c|c|c|c|}
\hline $\begin{array}{c}\text { Autor, año } \\
\text { publicación, país }\end{array}$ & Programa & Grupo control & $\begin{array}{l}\text { Tamaño de la } \\
\text { muestra, } \\
\text { diagnósticos }\end{array}$ & Seguimiento & Resultados \\
\hline $\begin{array}{l}\text { Barnes et al., } 2015 \\
\text { Australia }\end{array}$ & $\begin{array}{l}\text { Programa web } \\
\text { online "Recovery } \\
\text { road for bipolar } \\
\text { disorder" }\end{array}$ & $\begin{array}{l}\text { Webs "healthy li- } \\
\text { festyles" }\end{array}$ & $\begin{array}{l}233 \\
\mathrm{~TB} \mathrm{I} / \mathrm{II}\end{array}$ & 12 meses & $\begin{array}{l}\text { No diferencias significativas en re- } \\
\text { currencias }\end{array}$ \\
\hline $\begin{array}{l}\text { Depp et al., } 2012 \\
\text { USA }\end{array}$ & $\begin{array}{l}\text { Automonitoriza- } \\
\text { ción a través de } \\
\text { Smartphone }\end{array}$ & $\begin{array}{l}\text { Automonitoriza- } \\
\text { ción en papel y lá- } \\
\text { piz }\end{array}$ & $\begin{array}{l}40 \\
\mathrm{~TB}\end{array}$ & 12 semanas & $\begin{array}{l}\text { Significativamente menor cumpli- } \\
\text { mentación con smarthphone }\end{array}$ \\
\hline $\begin{array}{l}\text { Depp et al., } 2015 \\
\text { USA }\end{array}$ & $\begin{array}{l}\text { Automonitoriza- } \\
\text { ción a través de } \\
\text { smartphone: Pro- } \\
\text { grama PRISM }\end{array}$ & $\begin{array}{l}\text { Automonitoriza- } \\
\text { ción en papel y lá- } \\
\text { piz }\end{array}$ & $\begin{array}{l}82 \\
\text { TB }\end{array}$ & 24 semanas & $\begin{array}{l}\text { Reducción significativa en la MA- } \\
\text { DRS semanas } 6 \text { y } 12 \text { que se extin- } \\
\text { guió a las } 24 \text { semanas }\end{array}$ \\
\hline $\begin{array}{l}\text { Faurholt-Jepsen et } \\
\text { al., } 2015 \\
\text { USA }\end{array}$ & $\begin{array}{l}\text { Automonitoriza- } \\
\text { ción a través de } \\
\text { Smartphone: Pro- } \\
\text { grama MONAR- } \\
\text { CA }\end{array}$ & $\begin{array}{l}\text { Placebo y trata- } \\
\text { miento habitual }\end{array}$ & $\begin{array}{l}78 \\
\mathrm{~TB}\end{array}$ & 6 meses & $\begin{array}{l}\text { Adecuada correlación entre datos } \\
\text { objetivos registrados en el smar- } \\
\text { tphone y sintomatología depresiva } \\
\text { omaníaca }\end{array}$ \\
\hline
\end{tabular}

Nota . MADRS= Escala Montgomery Asberg de depresión; Programa PRISM: Personalized Real.Time Intervention for Stabilizing Mood: Programa MONARCA (MONitoring, treAtment and pRediCtion of bipolar disorder episodes); TB: Trastorno Bipolar; TB I: Trastorno Bipolar tipo I; TB II: Trastorno Bipolar tipo II.

y mental (Burns y Mohr, 2013). Se han usado para implementar estrategias que incluyen autovaloraciones, psicoeducación, fijación de objetivos, desarrollo de habilidades y técnicas de feedback. Utilizan tecnología que incluye teléfonos y videoconferencias, intervenciones a través de la web, uso de dispositivos móviles, sensores para la monitorización de pacientes, medios de comunicación social, realidad virtual y juegos para 
educar en salud (Mohr, Burns, Schueller, Clarke, y Klinkman, 2013).

Intervenciones previas con tecnología electrónica en el trastorno bipolar

Programa PRISM (Personalized Real-Time Intervention for Stabilizing Mood): Se utiliza una PDA que almacena los datos directamente sin una intervención directa con los terapeutas (Depp et al., 2010). Se basa en una técnica de intervención psicoeducativa breve para el trastorno bipolar (Bauer, 2003). Los participantes reciben previamente dos sesiones para entrenarse en la identificación de los síntomas más típicos de depresión y manía y sus pródromos, a la vez que se identifican respuestas adaptativas ante los mismos. Durante la intervención, contestan a una serie de preguntas acerca de su estado anímico y posibles pródromos, de modo similar a cómo lo harían mediante un gráfico vital. Cuando se detectan síntomas prodrómicos, en la pantalla de la PDA aparece una estrategia de afrontamiento. Esta intervención posibilita que se puedan personalizar estrategias y signos tempranos de recaída en tiempo real. Un ensayo aleatorizado controlado demostró que este programa mejora el impacto de la psicoeducación sobre los síntomas depresivos. Sin embargo, los beneficios se ven limitados cuando se interrumpe (Depp et al., 2015).

Programa FIMM (Facilitated Integrated Mood Management): Consiste en un tratamiento psicoeducativo que comprende cinco sesiones. Combina tratamientos psicosociales basados en la evidencia, con la terapia farmacológica e incorpora un programa de monitorización basado en mensajes de móviles o mails. El sistema envía diaria o semanalmente mensajes de texto o e-mails a los participantes para completar autopuntuaciones en una escala de depresión y una de manía, junto con el sueño. Permite observar fluctuaciones y personalizar cambios de tratamiento o variaciones en el estilo de vida. En el estudio piloto de breve duración que incluyó a pacientes con trastorno bipolar tipo I y II, no se observó un cambio significativo a nivel sintomático, pero la trayectoria de los pacientes con síntomas depresivos evolucionó hacia la mejoría. Sí mejoraron de forma significativa las estrategias de manejo de los cambios de humor entre la primera y última semanas de seguimiento (Miklowitz et al., 2012).

Proyecto MONARCA (MONitoring, treAtment and pRediCtion of bipolar disorder episodes) (Faurholt-Jepsen et al., 2013): Es uno de los primeros ejemplos del uso de la monitorización móvil de carácter persuasivo, actualmente en proceso de verificación de eficacia y efi- ciencia. Consta de una aplicación Android diseñada para el trastorno bipolar. Permite introducir y recopilar datos de autoevaluación diariamente mediante una alarma. Consta de una plataforma web personalizada de acceso para pacientes, médicos y familias, que ofrece información gráfica y facilita la atención inmediata al paciente. Facilita la identificación de signos tempranos de recaída y un feedback interactivo con un grupo de intervención a través de una página personal, en la que se monitorizan los ítems de forma gráfica. Mediante el software se pueden monitorizar aspectos subjetivos (humor, irritabilidad, sueño) y objetivos (minutos de conversación por 24 horas en el Smartphone o mensajes de texto), actividad social ( $\mathrm{n}^{\mathrm{o}}$ de salidas o llamadas) o actividad física mediante un GPS o acelerómetro, junto con la adherencia al tratamiento. Permite una visión histórica de los datos cada semana para fomentar la conciencia por parte del paciente y apoyo psicoterapéutico a través del refuerzo cotidiano e intercambio de datos entre paciente y médico. Se trata de identificar marcadores biológicos, para detectar signos tempranos de un episodio.

A través de un estudio controlado con placebo y aleatorizado de grupos paralelos se demostró que algunos de los datos objetivos recogidos se correlacionan con síntomas del trastorno bipolar, por ejemplo la inactividad física con una mayor prevalencia de síntomas depresivos o bien un descenso en el número de llamadas. No sucedió así con los síntomas maníacos (Faurholt-Jepsen et al., 2014). Esta mayor dificultad a la hora de autovalorar los síntomas maníacos se relaciona con las variaciones del insight en función de la fase que atraviesa el paciente, viéndose reducida en las fases maníacas (Depp et al., 2014). Se plantea así el potencial de los datos registrados en el Smartphone como biomarcadores del grado de actividad en función de la fase afectiva en pacientes bipolares (Faurholt-Jepsen et al., 2015).

Programa IABD (Improving Adherence in Bipolar Disorder): Diseñado para mejorar la adherencia tanto al tratamiento farmacológico como psicoterapéutico. Remite avisos diarios sobre citas, tratamiento e información sobre estado de ánimo, ansiedad, necesidad de tomar tratamiento o preocupación sobre efectos secundarios y reenvía dicha información, obteniendo un feedback en caso necesario. En un estudio piloto se encontró que la sintomatología depresiva se reducía significativamente a lo largo del periodo de estudio y las tasas de adherencia al tratamiento eran relativamente altas (Wenze, Armey y Miller, 2014).

Programa DERROTA (Beating Bipolar): Se trata de un programa de psicoeducación on line para personas con 
trastorno bipolar en temas como conciencia de enfermedad, adherencia al tratamiento, detección temprana de crisis o estilo de vida organizado y sano. Ha demostrado su buena aceptación por los pacientes, con una mayor utilidad especialmente en aquellos recientemente diagnosticados (Poole, Simpson y Smith, 2012). En un ensayo aleatorizado se encontró un modesto efecto en la mejora de la calidad de vida de los pacientes (Smith et al., 2011).

Sistema ChronoRecord: Este sistema consiste en un software instalado en un ordenador que recoge el humor, medicación y sueño desde el domicilio del paciente. Ha demostrado validez entre las autopuntuaciones en el registro del paciente y las puntuaciones del clínico en la Escala de Depresión de Hamilton (HAMD) (Bauer et al., 2004) y en la de manía de Young (YMRS) (Bauer et al., 2008).

App eMoods Bipolar MoodTracker2: Es un método de autovaloración del estado mental. El sistema permite introducir puntuaciones diarias del estado anímico y los monitoriza de forma electrónica. El paciente también señala las horas de sueño, nivel de ansiedad o la toma de medicación diariamente y la información puede ser compartida con un familiar, cuidador o clínico. El programa envía un informe mensual al paciente y al clínico con las puntuaciones.

App OPTIMISMO3 y TrueColors4: Ambas son Apps desarrolladas para la autovaloración por parte del paciente del humor, actividades y calidad de sueño. El objetivo es la monitorización de la depresión y de los cambios anímicos.

SMS: Otras intervenciones han consistido en utilizar mensajes de texto que los pacientes responden semanal- mente para monitorizar e identificar síntomas tempranos de recaída y así poder intervenir precozmente. En un estudio prospectivo que incluyó 62 pacientes con trastorno bipolar tipo I y II, se encontró que los resultados eran similares a los descritos con métodos tradicionales y suponían una alternativa válida de monitorización del humor (Bopp et al., 2010).

Técnicas de reconocimiento automático del humor: Utilizan aplicaciones móviles con sensores y modelos, que tratan de predecir el estado anímico de los pacientes. Se recogen datos objetivos y subjetivos de forma autoaplicada para tratar de estimar patrones futuros (Frost, Doryab, Faurholt-Jepsen, Kessing y Bardram, 2013). Se basan en el hallazgo de que la cuantificación objetiva de datos a través de medidas fisiológicas o índices de actividad, puede diferir notablemente de la percepción subjetiva a través de los autorregistros de los pacientes (Bussmann, Ebner-Priemer y Fahrenberg, 2009). Utilizan un sensor que aporta datos objetivos (acelerómetros y sistemas de posicionamiento global, detectores de luz y movimiento, hábitos alimentarios y nocturnos o llamadas telefónicas), entre otros (Prociow, Wac y Crowe, 2012). En un trabajo reciente se describe un sistema, basado en sensores en el Smartphone capaz de reconocer estados depresivos y maníacos y sus cambios de la forma más objetiva posible. Para ello analizan una base de datos real de 10 pacientes que recoge un periodo de 12 semanas midiendo aspectos como la interacción social, actividad física y rutinas diarias para optimizar la detección de los cambios, es decir aumento o descenso de estas actividades, en función de la presencia de una fase maníaca o depresiva (Grunerbl et al., 2015).

Las intervenciones previas figuran en la Tabla 3.

Tabla 3. Intervenciones con tecnología electrónica en el Trastorno bipolar

\author{
Programa PRISM (Personalized Real-Time Intervention for Stabilizing Mood) (Depp et al., 2010) \\ Programa FIMM (Facilitated Integrated Mood Management (Miklowitz et al., 2012) \\ Proyecto MONARCA (MONitoring, treAtment and pRediCtion of bipolar disorder episodes) (Faurholt-Jepsen et al., 2013) \\ Programa IABD (Improving Adherence in Bipolar Disorder) (Wenze et al., 2014) \\ Programa DERROTA (Beating Bipolar) (Smith et al., 2011) \\ SistemaChronoRecord (Bauer et al., 2004) \\ App eMoods Bipolar Mood Tracker² \\ App OPTIMISMO ${ }^{3}$ \\ App TrueColors ${ }^{4}$
}




\section{Discusión}

Aunque el potencial de las TIC en el terreno de las enfermedades mentales está todavía por determinar, su desarrollo se ha considerado un aspecto crucial para la promoción de la salud (Botella et al., 2012). Revisiones previas han concluido que las intervenciones guiadas a través de Internet son efectivas en la depresión y su eficacia no es inferior a los tratamientos "face to face" (Cuijpers y Riper, 2014). En cuanto a las ventajas de su utilización en el trastorno bipolar, hay que considerar que en esta enfermedad los pródromos suelen manifestarse a través de cambios, generalmente sutiles, a nivel conductual. Una de las principales razones por las que disminuye la eficacia de la intervención facultativa, tanto médica como psicológica, es el retraso en la intervención prodrómica. Como hemos señalado, este tipo de registros permiten una detección precoz de síntomas de inicio y algunos de ellos, incorporan nuevas tecnologías a través de sensores para la detección precisa de aspectos de gran interés como la actividad motora, los movimientos repetitivos durante los episodios maníacos o el retardo psicomotor en la depresión (Vancampfort et al., 2013). De hecho, hay trabajos que confirman la correlación entre los niveles de actividad física y la evaluación de la depresión mediante escalas, con una mayor precisión al considerar intervalos específicos del día en comparación con el nivel de actividad física durante todo el día (Osmani, 2013). Además, dado que el insight en el trastorno bipolar, se ve generalmente reducido en momentos de empeoramiento clínico, especialmente maníaco o hipomaníaco (De Assis da Silva et al., 2015), las nuevas tecnologías pueden ayudar a medir síntomas más objetivos que se ven afectados durante los episodios, como la actividad física o social (Faurholt-Jepsen et al., 2014). Un reciente trabajo en el que se monitorizó a través del Smartphone el estado anímico, actividad física e interacción social en un pequeño grupo de pacientes diagnosticados de trastorno bipolar concluyó que los síntomas depresivos eran predichos por un descenso en la interacción social (menor número de mensajes de texto) y en la actividad física. En cuanto a la sintomatología maníaca, se correlacionaba significativamente con la mayor tendencia a la sociabilidad pero no por cambios objetivos como la actividad física, aunque este hallazgo podría ser un sesgo inherente a la escasa prevalencia de síntomas maníacos en la muestra (Beiwinkel et al., 2016).

En el aspecto temporal, la capacidad de conocer los comportamientos y cambios clínicos (sueño, humor...) en tiempo real que ofrecen los móviles, permite una intervención más precoz y un beneficio en personas con potencial riesgo suicida (Clough y Casey, 2011).
En cuanto a la aceptación por parte de los pacientes, existen estudios cualitativos de feedback que sugieren que son conscientes de los beneficios de este tipo de técnicas y de su integración en la rutina clínica diaria (Palmier-Claus et al., 2013). En el trastorno bipolar, se describe un aumento en la creatividad, que les hace proclives a aceptar nuevos enfoques terapéuticos (Santosa et al., 2007) y a adaptarse rápidamente a estos nuevos sistemas de recogida de información con unos resultados fiables (Bopp et al., 2010). De hecho, en un estudio se analizaba la aceptabilidad de una intervención de dos semanas de duración llevada a cabo mediante PDAs en un grupo de pacientes con trastorno bipolar. Los participantes completaban unas evaluaciones dos veces al día sobre los síntomas y potenciales factores de riesgo de no adherencia y recibían de forma automática y semiindividualizada un feedback basado en sus respuestas. Los resultados fueron muy positivos, los pacientes señalaban que este método les había enseñado a conocer mejor su estado anímico y otros síntomas, así como a mejorar la adherencia al tratamiento y como un estímulo para contactar con su médico cuando era preciso (Wenze et al., 2014).

Con el objetivo de comparar la adaptación de los pacientes a los métodos tradicionales de recogida de información y a las nuevas tecnologías, Schärer et al adaptaron el National Institute of Mental Health Prospective Life-Chart para su uso en ordenador. Los pacientes señalaron que preferían estos dispositivos a los gráficos vitales en papel y lápiz, fundamentalmente porque percibían menos estigma social (Scharer et al., 2002). En un ensayo aleatorizado los pacientes tras recibir cuatro sesiones psicoeducativas, eran asignados durante diez semanas bien al Programa PRISM descrito anteriormente (Depp et al., 2010) o bien a una monitorización a través de un registro en lápiz y papel. Las tasas de retención fueron elevadas y similares y ambas estrategias se asociaban con una alta satisfacción. La reducción de la sintomatología depresiva era significativamente más elevada con el uso de móviles. Sin embargo estos efectos se extinguían a lo largo de las 24 semanas de seguimiento (Depp et al., 2015). Otros estudios que examinan la validez de la utilización del gráfico vital integrado en una App, encuentran que dichas puntuaciones predicen la gravedad de los síntomas depresivos de un modo muy certero (Scharer, Krienke, Graf, Meltzer y Langosch, 2015). También se ha analizado si existe una correlación adecuada entre los autorregistros electrónicos de los pacientes mediante ordenadores y las puntuaciones de entrevistadores entrenados. Programas como el ACASI (Audio Computer-Assisted Self-Interviewing) que se aplica en trastornos mentales graves (Chinman, Young, Schell, Hassell y Mintz, 2004), encontraron una correlación elevada. 
Otro aspecto a tener en cuenta es la adherencia terapéutica. Las TIC han demostrado su eficacia en la mejora de la cumplimentación terapéutica en un gran número de enfermedades médicas como la diabetes o el VIH, entre otras (Lewis et al., 2013; Mulvaney, Anders, Smith, Pittel y Johnson, 2012). En el trastorno bipolar, el incumplimiento terapéutico tiene una gran relevancia y afecta a todas las fases de la enfermedad (Colom et al., 2000). Sin embargo, escasos trabajos analizan la influencia de las TIC en la adherencia. Entre las ventajas señaladas por los pacientes figuran la ayuda de los recordatorios de citas y toma de fármacos o la colaboración en la instauración de rutinas beneficiosas para la enfermedad. En cambio, la tendencia a la distraibilidad, desmotivación o la falta de insight podrían afectar negativamente a la adherencia a las propias TIC. Pese a ello, las tasas de cumplimentación de las sesiones por parte de esta población (Wenze et al., 20014) son superiores a las encontradas con muestras no clínicas (Wenze, Gunthert y German, 2012). Otra ventaja es una mayor accesibilidad ya que este tipo de técnicas superan las barreras del transporte, descrito como un motivo de menor participación en las intervenciones psicosociales (Kilbourne, 2005).

En términos de farmacoeconomía se trata un método barato y accesible, que aporta una mayor flexibilidad y comodidad para el usuario y que facilita el acceso a la salud a poblaciones dispersas y remotas y a personas con dificultades de movilidad. Pueden ayudar a reducir la frecuencia o duración de las intervenciones, alargar el efecto de algunas sesiones o consultas mediante recordatorios (Wenze et al., 2014) Todo esto se podría reflejar en una reducción de gastos.

En cuanto a las desventajas que pueden presentar las TIC, los mismos problemas que aparecen en la cumplimentación farmacológica nos los podemos encontrar en este tipo de aplicaciones. Entre ellos, el abandono en la cumplimentación o las respuestas al azar (Shiffman, Stone y Hufford, 2008). Además el hecho de aumentar la complejidad de los sistemas para obtener más información, puede reducir la adherencia de los pacientes. Algunos trabajos encuentran una reducción progresiva en las tasas de cumplimentación superior en el caso de los móviles, que con los cuestionarios habituales en papel. Sin embargo las puntuaciones en los móviles en manía y depresión se han asociado significativamente con las puntuaciones de los clínicos, no siendo así en el caso de las puntuaciones en papel. Este último hallazgo puede justificarse en función de uno de los principales sesgos de los cuestionarios en papel que es la cumplimentación "aleatoria", o bien retrospectiva de varios de ellos, mientras que con las nuevas tecnologías la cumplimentación es prospectiva (Depp, Kim, de Dios, Wang y Ceglowski, 2012).
También se ha señalado que en términos de la aceptabilidad, los sistemas electrónicos pueden generar un distanciamiento físico y emocional entre el terapeuta y el paciente y por tanto, ir en detrimento del efecto beneficioso de una buena alianza terapéutica (Martin, Garske y Davis, 2000) y de la comunicación no verbal.

Según los resultados del estudio Monarca presentado previamente, las fluctuaciones en el estado anímico tienen una influencia directa a la hora de cumplimentar los cuestionarios. Esta mayor dificultad para autovalorar los síntomas maníacos, se relaciona con las variaciones del insight en función de la fase que atraviesa el paciente (Depp et al., 2014), aunque esta sería una desventaja presente también en los cuestionarios en papel.

Por otro lado, las TIC no garantizan en el momento actual la equidad en la atención a todos los pacientes ya que la accesibilidad se ve reducida en sustratos socioeconómicos más bajos, poblaciones analfabetas tecnológicamente o pacientes de mayor edad con menos conocimientos informáticos. Además es importante considerar que existen profesionales reacios a su uso. En general aquellos que ejercen en poblaciones rurales tienden a reconocer unos mayores índices de satisfacción (Menon et al., 2001).

Uno de los problemas detectados por el Informe EIU $^{1}$, señalado por el $53 \%$ de los encuestados era el temor a que los pacientes malinterpretaran sus datos y tomaran decisiones erróneas. También se plantean problemas médico legales que tienen que ver con la protección de datos o confidencialidad, mantenimiento del secreto profesional y la administración del consentimiento informado. En dicho informe, se cuestionaba si era posible asegurar a los pacientes que sus datos estaban seguros. La mitad de los encuestados consideraban que la cautela de los pacientes acerca de la posible violación de su privacidad podría ser un obstáculo para la adopción de la $\mathrm{m}$-health. En líneas generales hay que considerar que se trata de cuidados de salud mental, que requieren una evaluación de la seguridad y claridad cuidadosa.

A nivel técnico, también se han planteado algunas limitaciones como el tamaño de la pantalla, el número máximo de caracteres disponibles, las dificultades de protocolos largos o complejos, el agotamiento de los participantes y la velocidad de procesamiento del software que afecta necesariamente al diseño del estudio. En cuanto a los aspectos económicos, también la programación de los sistemas móviles y el desarrollo de entrenamiento y protocolos suponen un coste en tiempo que habrá que comparar con los potenciales ahorros.

En la presente revisión, que cuenta con la limitación de no ser sistemática, se han detectado una serie de aspectos a mejorar en los estudios analizados. Las mues- 
tras de los trabajos son pequeñas, destaca la escasez de ensayos aleatorizados controlados y existen sesgos como la compensación económica a los participantes en algunos trabajos, que podría aumentar las tasas de adherencia (Wenze et al., 2014), la participación de pacientes más motivados, con más conciencia de enfermedad y más capacitados para usar las herramientas electrónicas de automonitorización (Faurholt-Jepsen, Munkhom, Frost, Bardram y Kessing, 2016). En el caso de las intervenciones basadas en el empleo de mensajes de móviles, han sido criticadas por ser demasiado simples, no incluir un feedback bidireccional entre pacientes y médicos y no disponer de datos electrónicos basados en medidas objetivas de la psicopatología afectiva (Bopp et al., 2010).

\section{Conclusiones}

Como hemos visto en esta revisión, las intervenciones a través de las TIC tratan de desarrollar recomendaciones clínicas y tratamientos que los pacientes puedan incorporar a sus rutinas diarias, en los medios en los que se mueven habitualmente. Las TIC permiten recibir apoyo adicional entre visitas, adaptando las sesiones y el seguimiento en función de las necesidades o demandas del paciente y practicar con herramientas novedosas, aplicando nuevas terapias in vivo. Se han considerado un método de bajo coste, fácilmente difundible para incrementar la frecuencia y la duración del contacto terapéutico (Wenze et al., 2014).

Aunque los estudios que analizan la utilización de las TIC en el trastorno bipolar son escasos, existen datos positivos tanto en lo que respecta a la adhesión de los pacientes a estas técnicas con pequeñas pérdidas durante el seguimiento (Bauer, 2005), como en cuanto a elevada correlación entre las puntuaciones entre clínicos y pacientes (Chinman et al., 2004).

Sin embargo no están exentas de críticas, dado que los pacientes podrían no ser completamente sinceros en sus contestaciones y los que colaboran, podrían constituir un sesgo en el sentido de ser más cumplimentadores y con mejor conciencia de enfermedad. Algunos ensayos aleatorizados controlados basados en intervenciones psicológicas a través de la web encuentran resultados negativos, pero cuentan con numerosas limitaciones que tendrán que ser perfeccionadas en el futuro (Barnes, Hadzi-Pavlovic, Wilhelm y Mitchell, 2014).

En el futuro se plantean varias líneas de investigación. Por ejemplo, no sabemos si la adopción y cumplimentación será satisfactoria en todos los grupos de edad, clase socioeconómica, países, diferentes comorbilidades o niveles de gravedad de enfermedad o cronicidad (Miklowitz et al., 2012). Su aplicación en población adoles- cente bipolar es especialmente interesante dada la familiaridad que presentan en su uso y la importancia de la psicoeducación en este grupo. También está pendiente estudiar la incorporación de los familiares al uso de estas tecnologías. En algunos programas se involucra a los familiares en la identificación de pródromos y en el desarrollo de estrategias alternativas ante las recaídas (Miklowitz et al., 2012), ya que éstos pueden detectar los pródromos más rápida y acertadamente que los propios pacientes (Reinares et al., 2008). Por otra parte, es muy poco probable que los pacientes sigan manteniendo contacto semanal infinitamente. Una dirección es el desarrollo de enfoques escalonados en los que las intervenciones puedan aumentar en frecuencia o ser más interactivas durante periodos críticos (Depp et al., 2010).

También surgen dilemas éticos en lo que respecta a la confidencialidad. El mantenimiento de ésta se puede mejorar añadiendo passwords de identificación y encriptación. En general hay que optimizar la seguridad en la protección de los datos.

En resumen, aunque la aplicación de las TIC en el trastorno bipolar parece prometedora, para que se pueda apoyar su uso de una forma más generalizada, es necesario realizar más estudios basados en ensayos clínicos con un diseño y control adecuados. Es precisa la inclusión de grupos de pacientes con características clínicas y demográficas homogéneas, así como más estudios comparativos que avalen su eficacia y eficiencia. También es importante analizar las características delos pacientes excluidos y las razones por las que declinan su participación, para conocer las posibilidades futuras de generalización de estas técnicas. Se deben conocer los requerimientos económicos del desarrollo y mantenimiento de las TIC empleadas, estos datos son necesarios para avalar su implementación clínica. En definitiva, son precisos nuevos estudios que analicen los efectos tanto beneficiosos como perjudiciales de las TIC en el trastorno bipolar.

\section{Referencias}

Barnes, C. W., Hadzi-Pavlovic, D., Wilhelm, K., \& Mitchell, P. B. (2014). A web-based preventive intervention program for bipolar disorder: Outcome of a 12-months randomized controlled trial. Journal of Affective Disorders, 174, 485-492.

Bauer, M., Grof, P., Rasgon, N., Gyula, L., Glenn, T., \& Whybrow P. (2005). New computer based tool for the longitudinal study of bipolar disorder. Aspects of Affect, 2, 101-108.

Bauer, M. (2003). Structured group therapy for bipolar disorder: The Life Goals Program. New York: Springer.

Bauer, M., Grof, P., Gyulai, L., Rasgon, N., Glenn, T., \& Whybrow, P. C. (2004). Using technology to improve longitudinal studies: self-reporting with ChronoRecord in bipolar disorder. Bipolar Disorders, 6, 67-74. 
Bauer, M., Wilson, T., Neuhaus, K., Sasse, J., Pfennig, A., Lewitzka, U., \& Whybrow, P. C. (2008). Self-reporting software for bipolar disorder: validation of ChronoRecord by patients with mania. Psychiatry Research, 159(3), 359-366.

Beiwinkel, T., Kindermann, S., Maier, A., Kerl, C., Moock, J., Barbian, G., \& Rossler, W. (2016). Using Smartphones to Monitor Bipolar Disorder Symptoms: A Pilot Study. Journal of Medical Internet Research, 3(1), e2.

Bird, K. T. (1975). Telemedicine: concept and practice. Springfield, Illinois: Thomas.

Bopp, J. M., Miklowitz, D. J., Goodwin, G. M., Stevens, W., Rendell, J. M., \& Geddes, J. R. (2010). The longitudinal course of bipolar disorder as revealed through weekly text messaging: a feasibility study. Bipolar Disorders, 12(3), 327-334.

Botella, C., Riva, G., Gaggioli, A., Wiederhold, B. K., Alcaniz, M., \& Banos, R. M. (2012). The present and future of positive technologies. Cyberpsychology, Behavior, and Social Networking, 15(2), 78-84.

Burns, \& Mohr, D. C. (2013). eHealth and Behavioral Intervention Technologies. Encyclopedia of Behavioral Medicine (pp. 659-664). New York: Springer.

Burns, J. M., Davenport, T. A., Durkin, L. A., Luscombe, G. M., \& Hickie, I. B. (2010). The internet as a setting for mental health service utilisation by young people. Medical Journal of Australia, 192(11 Suppl), S22-26.

Bussmann, J. B., Ebner-Priemer, U. W., \& Fahrenberg, J. (2009). Ambulatory activity monitoring: Progress in measurement of activity, posture, and specific motion patterns in daily life. European Psychologist, 14(2), 142-152.

Cipresso, P., Serino, S., Villani D., Repetto C., Selitti L., Albani G., Mauro A., Gaggioli A., \& Riva, G. (2012). Is your phone so smart to affect your states? An exploratory study based on psychophysiological measures. Neurocomputing, 84, 23-30.

Clough, B. A., \& Casey, L.M. (2011).Technological adjuncts to increase adherence to therapy: A review. Clinical Psychology Review, 31, 697- 710.

Cohn, M. (2012). Hopkins researchers aim to uncover which mobile health applications work. Mar 14 The Baltimore Sun. http://articles.baltimoresun.com/2012-03-14/health/bs-hsmobilehealth-apps-20120314_1_health-apps-mhealth-mobile-health

Colom, F., Vieta, E., Martinez-Aran, A., Reinares, M., Benabarre, A., \& Gasto, C. (2000). Clinical factors associated with treatment noncompliance in euthymic bipolar patients. Journal of Clinical Psychiatry, 61(8), 549-555.

Cuijpers, P., \& Riper, H. (2014). Internet interventions for depressive disorders: an overview. Revista de Psicopatología y Psicología Clínica, 19(3), 209-216.

Chinman, M., Young, A. S., Schell, T., Hassell, J., \& Mintz, J. (2004). Computer-assisted self-assessment in persons with severe mental illness. Journal of Clinical Psychiatry, 65(10), 1343-1351.

De Assis da Silva, R., Mograbi, D. C., Silveira, L. A., Nunes, A. L., Novis, F. D., Landeira-Fernandez, J., \& Cheniaux, E. (2015). Insight Across the Different Mood States of Bipolar Disorder. Psychiatric Quarterly, 86(3), 395-405

Depp, C. A., Ceglowski, J., Wang, V. C., Yaghouti, F., Mausbach, B. T., Thompson, W. K., \& Granholm, E. L. (2015). Augmenting psychoeducation with a mobile intervention for bipolar disorder: a randomized controlled trial. Journal of Affective Disorders, 174, 23-30.
Depp, C. A., Harmell, A. L., Savla, G. N., Mausbach, B. T., Jeste, D. V., \& Palmer, B. W. (2014). A prospective study of the trajectories of clinical insight, affective symptoms, and cognitive ability in bipolar disorder. Journal of Affective Disorders, 152154, 250-255.

Depp, C. A., Kim, D. H., de Dios, L. V., Wang, V., \& Ceglowski, J. (2012). A Pilot Study of Mood Ratings Captured by Mobile Phone Versus Paper-and-Pencil Mood Charts in Bipolar Disorder. Journal of Dual Diagnosis, 8(4), 326-332.

Depp, C. A., Mausbach, B., Granholm, E., Cardenas, V., BenZeev, D., Patterson, T. L., Lebowitz, B. D., \& Jeste, D. V. (2010). Mobile interventions for severe mental illness: design and preliminary data from three approaches. The Journal of Nervous and Mental Disease, 198(10), 715-721.

Faurholt-Jepsen, M., Frost, M., Vinberg, M., Christensen, E. M., Bardram, J. E., \& Kessing, L. V. (2014). Smartphone data as objective measures of bipolar disorder symptoms. Psychiatry Research, 217(1-2), 124-127.

Faurholt-Jepsen, M., Munkholm, K., Frost, M., Bardram, J. E., \& Kessing, L. V. (2016). Electronic self-monitoring of mood using IT platforms in adult patients with bipolar disorder: A systematic review of the validity and evidence. BMC Psychiatry, 16(1), 7.

Faurholt-Jepsen, M., Vinberg, M., Christensen, E. M., Frost, M., Bardram, J., \& Kessing, L. V. (2013). Daily electronic self-monitoring of subjective and objective symptoms in bipolar disorder--the MONARCA trial protocol (MONitoring, treAtment and pRediCtion of bipolAr disorder episodes): A randomised controlled single-blind trial. BMJ Open, 3(7).

Faurholt-Jepsen, M., Vinberg, M., Frost, M., Christensen, E. M., Bardram, J., \& Kessing, L. V. (2015). Smartphone data as an electronic biomarker of illness activity in bipolar disorder. $\mathrm{Bi}$ polar Disorder, 17, 715-728.

Faurholt-Jepsen, M., Vinberg, M., Frost, M., Christensen, E., Bardram, J., \& Kessing, L. (2014). Daily electronic monitoring of subjective and objective measures of illness activity in bipolar disorder using smartphones inverted question mark the MONARCA II trial protocol: a randomized controlled single-blind parallel-group trial. BMC Psychiatry, 14(1), 309.

FDA. (2015). Mobile Medical Applications Guidance for Industry and Food and Drug Administration Staff Retrieved from http:// www.fda.gov/downloads/MedicalDevices/DeviceRegulationandGuidance/GuidanceDocuments/UCM263366.pdf? source=govdelivery\&utm_medium=email\&utm_source=govdelivery.

Frost, M., Doryab, A., Faurholt-Jepsen, M., Kessing, L. V., \& Bardram, J. E. (2013). Supporting disease insight through data analysis: Refinements of the monarca self-assessment system. Proceedings of UbiComp, 133-142.

Goodwin, G. M. (2009). Evidence-based guidelines for treating bipolar disorder: revised second edition--recommendations from the British Association for Psychopharmacology. Journal of Psychopharmacology, 234, 346-388.

Grunerbl, A., Muaremi, A., Osmani, V., Bahle, G., Ohler, S., Troster, G., Mayora, O., Haring, C., \& Lukowicz, P. (2015). Smartphone-based recognition of States and state changes in bipolar disorder patients. IEEE Journal of Biomedical and Health Informatics, 19(1), 140-148.

Kilbourne, A. M. (2005). Bipolar disorder in late life: future directions in efficacy and effectiveness research. Current Psychiatry Reports, 7(1), 10-17. 
Kimhy, D., Delespaul, P., Corcoran, C., Ahn, H., Yale, S., \& Malaspina, D. (2006). Computerized experience sampling method (ESMc): assessing feasibility and validity among individuals with schizophrenia. Journal of Psychiatr Research, 40(3), 221-230.

Lewis, M. A., Uhrig, J. D., Bann, C. M., Harris, J. L., Furberg, R. D., Coomes, C., \& Kuhns, L. M. (2013). Tailored text messaging intervention for HIV adherence: a proof-of-concept study. Health Psychology, 32(3), 248-253.

Livianos, L., Pino, A., \& Sierra, P. (2003). Manual del gráfico del estado de ánimo para trastornos afectivos recurrentes. Madrid: Emisa.

Marangell, L. B., Dennehy, E. B., Miyahara, S., Wisniewski, S. R., Bauer, M. S., Rapaport, M. H., \& Allen, M. H. (2009). The functional impact of subsyndromal depressive symptoms in bipolar disorder: data from STEP-BD. Journal of Affective Disorders, 114(1-3), 58-67.

Martin, D. J., Garske, J. P., \& Davis, M. K. (2000). Relation of the therapeutic alliance with outcome and other variables: a meta-analytic review. Journal of Consulting and Clinical Psychology, 68(3), 438-450.

Menon, A. S., Kondapavalru, P., Krishna, P., Chrismer, J. B., Raskin, A., Hebel, J. R., \& Ruskin, P. E. (2001). Evaluation of a portable low cost videophone system in the assessment of depressive symptoms and cognitive function in elderly medically ill veterans. Journal of Nervous and Mental Disease, 189(6), 399-401.

Miklowitz, D. J., Price, J., Holmes, E. A., Rendell, J., Bell, S., Budge, K., \& Geddes, J. R. (2012). Facilitated Integrated Mood Management for adults with bipolar disorder. Bipolar Disorders, 14(2), 185-197.

Mohr, D. C., Burns, M. N., Schueller, S. M., Clarke, G., \& Klinkman, M. (2013). Behavioral intervention technologies: evidence review and recommendations for future research in mental health. General Hospital Psychiatry, 35(4), 332-338.

Mulvaney, S. A., Anders, S., Smith, A. K., Pittel, E. J., \& Johnson, K. B. (2012). A pilot test of a tailored mobile and web-based diabetes messaging system for adolescents. Journal of Telemedicine and Telecare, 18(2), 115-118.

OMS. (2015). Paper presented at the Cibersalud [Internet]. $58^{a}$. Asamblea Mundial de la Salud; del 16 al 25 de mayo(resolución WHA58.28). Ginebra (Suiza).

Osmani, V. A., Maxhuni, A., Gruenerbl, A., Lukowicz, P., Haring,C., \& Mayora, O. (2013). Monitoring activity of patients with bipolar disorder using smartphones. Paper presented at the Int. Conf. Adv. Mobile Comput. Multimedia, New York, NY, USA.

Palmier-Claus, J. E., Rogers, A., Ainsworth, J., Machin, M., Barrowclough, C., Laverty, L., \& Lewis, S. W. (2013). Integrating mobile-phone based assessment for psychosis into people's everyday lives and clinical care: a qualitative study. $B M C$ Psychiatry, 13, 34.

Poole, R., Simpson, S. A., \& Smith, D. J. (2012). Internet-based psychoeducation for bipolar disorder: a qualitative analysis of feasibility, acceptability and impact. BMC Psychiatry, 12, 139.

Price, M., Yuen, E. K., Goetter, E. M., Herbert, J. D., Forman, E. M., Acierno, R., \& Ruggiero, K. J. (2014). mHealth: a mechanism to deliver more accessible, more effective mental health care. Clinical Psychology \& Psychotherapy, 21(5), 427-436.

Prociow, P., Wac, K., \& Crowe, J. (2012). Mobile psychiatry: towards improving the care for bipolar disorder. International Journal of Mental Health System, 6(1), 5.

Reinares, M., Colom, F., Sanchez-Moreno, J., Torrent, C., Martinez-Aran, A., Comes, M., Goikolea, J.M., Benabarre, A., \& Vieta, E. (2008). Impact of caregiver group psychoeducation on the course and outcome of bipolar patients in remission: a randomized controlled trial. Bipolar Disorders, 10(4), 511-519.

Santosa, C. M., Strong, C. M., Nowakowska, C., Wang, P. W., Rennicke, C. M., \& Ketter, T. A. (2007). Enhanced creativity in bipolar disorder patients: a controlled study. Journal of Affective Disorders, 100(1-3), 31-39.

Scharer, L. O., Hartweg, V., Valerius, G., Graf, M., Hoern, M., Biedermann, C., \& Walden, J. (2002). Life charts on a palmtop computer: first results of a feasibility study with an electronic diary for bipolar patients. Bipolar Disorders, 4 Suppl 1, 107-108.

Scharer, L. O., Krienke, U. J., Graf, S. M., Meltzer, K., \& Langosch, J. M. (2015). Validation of life-charts documented with the personal life-chart app - a self-monitoring tool for bipolar disorder. BMC Psychiatry, 15, 49.

Serino, S., Cipresso, P., Gaggioli, A., Pallavicini, F., Cipresso, S. , Campanario, D., \& Rivas, G. (2014). Smartphone for self-management of psychological stress: a preliminary evaluation of positive technology app. Revista de Psicopatología y Psicología Clínica, 19(3), 253-260.

Shiffman, S., Stone, A. A., \& Hufford, M. R. (2008). Ecological momentary assessment. Annual Review of Clinical Psychology, 4, 1-32.

Simon, G. E., \& Ludman, E. J. (2009). It's time for disruptive innovation in psychotherapy. Lancet, 374(9690), 594-595.

Smith, D. J., Griffiths, E., Poole, R., di Florio, A., Barnes, E., Ke1ly, M. J., \& Simpson, S. (2011). Beating Bipolar: exploratory trial of a novel Internet-based psychoeducational treatment for bipolar disorder. Bipolar Disorders, 13(5-6), 571-577.

Smyth, J. M., Wonderlich, S. A., Heron, K. E., Sliwinski, M. J., Crosby, R. D., Mitchell, J. E., \& Engel, S. G. (2007). Daily and momentary mood and stress are associated with binge eating and vomiting in bulimia nervosa patients in the natural environment. Journal of Consulting and Clinical Psychology, 75(4), 629-638.

Trull, T. J., Solhan, M. B., Tragesser, S. L., Jahng, S., Wood, P. K., Piasecki, T. M., \& Watson, D. (2008). Affective instability: measuring a core feature of borderline personality disorder with ecological momentary assessment. Journal of Abnormal Psychology, 117(3), 647-661.

Vancampfort, D., Correll, C. U., Probst, M., Sienaert, P., Wyckaert, S., De Herdt, A., \& De Hert, M. (2013). A review of physical activity correlates in patients with bipolar disorder. Journal of Affective Disorders, 145(3), 285-291.

Wade, A. G. (2010). Use of the internet to assist in the treatment of depression and anxiety: a systematic review. Primary Care Companion Journal of Clinical Psychiatry, 12(4).

Wenze, S. J., Armey, M. F., \& Miller, I. W. (2014). Feasibility and Acceptability of a Mobile Intervention to Improve Treatment Adherence in Bipolar Disorder: A Pilot Study. Behavior Modification, 38(4), 497-515. 
Wenze, S. J., Gunthert, K. C., \& German, R. E. (2012). Biases in affective forecasting and recall in individuals with depression and anxiety symptoms. Personality and Social Psychology Bulletin, 38(7), 895-906.

\section{Referencias web}

1 The Economist Intelligence Unit. (2015). Power to the patient: How mobile technology is transforming healthcare. A report from The
Economist Intelligence Unit. Available: http://www.economistinsights.com/analysis/how-mobile-transforming-healthcare

2 Technologies. (2013). Emoods bipolar mood tracker (version 1.0)[mobile software application]. [Online]. Available: https:// play.google.com/store/apps,"

${ }^{3}$ Optimism. (2014, Jul.). Optimism apps. [Online]. Available: www.findingoptimism.com.

${ }^{4}$ True colours-improved management for people with bipolar disorder (2014). [Online]. Available: http://oxtext.psych.ox.ac.uk/. 